\title{
Erythrocyte Deformability in the Fetus, Preterm, and Term Neonate
}

\author{
OTWIN LINDERKAMP, MONIKA GÜNTNER, WOLFGANG HILTL, AND VIVIAN M. VARGAS \\ Department of Pediatrics, University of Heidelberg, Heidelberg, and Department of Pediatrics, University of \\ Munich, Munich, Federal Republic of Germany
}

\begin{abstract}
Deformability of red blood cells (RBC) is an important determinant of microcirculation, of oxygen transport and release to the tissues, and of RBC life span. Deformability of RBC from five fetuses, 20 preterm infants, 20 term neonates, and 20 adults was determined by direct microscopic observation of RBC subjected to shear stresses of 6 to $85 \mathrm{dyn} / \mathrm{cm}^{2}$ using a counter-rotating rheoscope. There was no significant difference in deformability among $\mathrm{RBC}$ from the fetuses, the preterm and term neonates, and the adults at any shear stress. More than $95 \%$ of fetal, neonatal, and adult RBC were capable of tanktread motion. Compared to adults, the frequency distribution of RBC deformability was slightly broader in the fetuses and neonates because of the presence of more highly and poorly deformable RBC. The increased number of rigid RBC may contribute to the shortened life span of fetal RBC. (Pediatr Res 20:93-96, 1986)
\end{abstract}

Abbreviation

RBC, red blood cell

For the premature infant, maintenance of an adequate tissue oxygen supply is a major problem. Oxygen transport to the tissues is dependent on effective blood flow and on rapid oxygen release from the RBC. Both effective blood flow and the rate of oxygen uptake are influenced by the ability of $\mathrm{RBC}$ to deform (i.e. to assume new shapes) (1-3). RBC deformability is essential at four levels: 1) adaptation to higher shear forces in large vessels with rapid flow, thereby lowering blood viscosity $(1) ; 2$ ) entry of the 8- $\mu \mathrm{m}$ diameter RBC into 3- to 4- $\mu \mathrm{m}$ diameter capillaries (4); 3 ) acceleration of oxygen uptake and release in capillaries (3); and 4) passage through narrow splenic slits, thereby escaping destruction by immunological mechanisms (5). Failure of the $\mathrm{RBC}$ to deform sufficiently can therefore result in disturbed large and small vessel circulation, in tissue hypoxia and in RBC damage.

Impaired microcirculation is probably involved in the development of serious complications in preterm infants (e.g. necrotizing enterocolitis) (6). Whether peculiar deformability properties of preterm infants' $\mathrm{RBC}$ play a role in the pathogenesis of such complications is unknown. Furthermore, RBC life span is shorter (7) and bilirubin production is higher (8) in preterm infants than in term neonates and adults. Correlations between shortened life span and rigidity of $\operatorname{RBC}(2,9)$, therefore, suggest that $\mathrm{RBC}$ deformability in preterm infants is decreased.

Received August 30, 1984; accepted August 19, 1985.

Reprints, O. Linderkamp, M.D., Universitäts-Kinderklinik Heidelberg, Im Neuenheimer Feld 150, D-6900 Heidelberg, Federal Republic of Germany.

This work was supported by the Deutsche Forschungsgemeinschaft (Heisenberg Grant Li 291/3 and Research Grant 291/4-1).
Studies on the deformability of $\mathrm{RBC}$ from full-term neonates gave conflicting results: neonatal and adult $\mathrm{RBC}$ show no difference in deformability when studied under defined shear forces by a rheoscope (10) or an ektacytometer (11). However, RBC from term neonates require higher suction pressures for complete aspiration into narrow micropipettes (12) and are less filterable (13-16) than adult RBC.

Little is known about the deformability of RBC from preterm infants. Buchan (15) studied filterability of RBC from preterm infants with gestational age of 34 to $37 \mathrm{wk}$ and measured lower filtration rates in preterm infants compared to term neonates. Coulombel et al. (11) used an ektacytometer to determine deformability of RBC from 11 neonates with 35 to $40 \mathrm{wk}$ of gestational age. They did not find a correlation between RBC deformability and gestational age. Studies on the deformability of $\mathrm{RBC}$ from fetuses and small preterm infants do not appear to exist in the literature.

In the present study, deformability of $\mathrm{RBC}$ from fetuses with 18 to $20 \mathrm{wk}$ and from preterm and term neonates with 24 to 41 wk of gestational age were measured by means of a rheoscope. Our results indicate no significant difference in deformability between $\mathrm{RBC}$ from fetuses, preterm infants, term neonates, and adults.

\section{MATERIALS AND METHODS}

The deformability measurements were performed on blood samples from five fetuses (18-20 wk of gestation), 20 preterm infants, 20 term neonates, and 20 adults. The study was approved by the Department of Pediatrics Human Subjects Research Committee. Informed consent was obtained from the mothers of the five fetuses. Heparinized blood from five fetuses was collected by heart puncture immediately after prostaglandin $E_{2}$-induced abortion for nongenetic reasons. For the 40 neonates, $10 \mathrm{ml}$ of blood was collected from the placenta into heparin $(5 \mathrm{IU} / \mathrm{ml})$ immediately after cord clamping prior to delivery of the placenta. Twenty of the newborns were healthy full-term infants with gestational age of 38 to 41 wk and birth weight of 3210 to 3630 g; 10 were preterm infants with gestational age of 24 to $30 \mathrm{wk}$ and birth weight of 760 to $1440 \mathrm{~g}$; and 10 had gestational age of 31 to $36 \mathrm{wk}$ and birth weight of 1370 to $2690 \mathrm{~g}$. The gestational age of each infant was derived from the maternal history and confirmed by clinical assessment of maturity. All infants had birth weight appropriate for gestational age 10th to 90th percentile according to unpublished Munich Growth Charts). Infants with malformations, erythroblastosis, diabetic mothers, hemorrhage, intrauterine asphyxia, and those delivered by cesarean section were excluded, as were twins and infants with proven infection or high risk of infection. Adult blood samples were collected from 20 healthy hospital personnel via venipuncture into heparin.

All measurements were made at room temperature $\left(22 \pm 1^{\circ}\right.$ C) within $2 \mathrm{~h}$ after collection. Whole blood was not centrifuged 
and washed prior to dilution in the appropriate suspending medium, since the amount of blood available for our studies was limited and neonatal RBC are sensitive to morphological transformation and mechanical damage $(11,12)$. A part of each blood sample was used for analysis of hematological indices and routine measurements. Five $\mu$ l of blood were diluted 1:50 (hematocrit about $1 \%)$ in phosphate buffered saline $\left(0.005 \mathrm{M} \mathrm{KH}_{2} \mathrm{PO}_{4}+\right.$ $\mathrm{Na}_{2} \mathrm{HPO}_{4}, 0.153 \mathrm{NaCl}, \mathrm{pH} 7.40,295 \mathrm{mosmol} / \mathrm{kg}$ ) containing 20 $\mathrm{g} / \mathrm{dl}$ of dextran T-70 (Pharmacia, Uppsala, Sweden). The viscosity of these dextran solutions was 21 centipoise. This increase in the suspending medium viscosity via dextran is necessary in order to achieve cellular deformation in the rheoscope $(1,2)$. It does not alter the mechanical properties of the RBC membrane (17).

The deformability of single $\mathrm{RBC}$ was observed and measured using a counter-rotating, cone-plate rheoscope $(1,2)$ (Effenberger, Munich, FRG) which was mounted on an inverted microscope (Diavert, Leitz, Wetzlar, FRG); details of the method have been described elsewhere (10). Six different shear stresses from 6 to $85 \mathrm{dyn} / \mathrm{cm}^{2}$ were applied and photomicrographs of the cells (Fig. 1) were taken at each of the shear stresses. Length (L) and width (W) of $40 \mathrm{RBC}$ were measured in each sample at each shear stress using a computerized micrometer system (MOPVideoplan, Kontron Electronics, Eching-Munich, FRG). The RBC deformation parameter $\mathrm{D}$ is defined as: $\mathrm{D}=(\mathrm{L}-\mathrm{W}) /$ $(\mathrm{L}+\mathrm{W})$. The percentage of tank-treading $\mathrm{RBC}$ at each shear stress was determined by microscopic inspection through the microscope $(1,18)$.

$\mathrm{D}$ values increase with increasing cellular deformation (Fig. 2) and approach a limiting value at high shear stresses. The value of $\mathrm{D}$ is zero in the absence of deformation. The rheoscope as a microrheological method allows the deformation of single RBC to be determined, whereas other methods (e.g. micropore filtration, viscometry, ektacytometer) only provide average information for a $\mathrm{RBC}$ population. The rheoscope is sensitive enough to detect slight alterations of RBC deformability for entire cell populations as well as the presence of a subpopulation of poorly deformable cells (2). The latter ability depends on the fraction of abnormal RBC and on the total number of cells studied.
$\mathrm{RBC}$ count, hemoglobin concentration and mean corpuscular volume were determined using a Coulter-Counter (Coulter Electronicy Inc., Harpenden, Herts, England). Hemoglobin F was quantified by the alkali denaturation technique (19).

Statistical analyses were performed to test for differences in measurements among the fetuses, the two groups of preterm infants, the full-term neonates and the adults (analysis of variance) (20). Frequency distributions of RBC deformation were established for the cells in each of the five groups at each shear stress. These frequency distributions were compared by constructing contingency tables and by using a $\chi^{2}$ test.

\section{RESULTS}

Table 1 presents hematological data and cellular deformation measurements at selected shear stresses for RBC from fetuses, preterm infants, term neonates, and adults. Mean corpuscular volume, mean corpuscular hemoglobin, and hemoglobin $\mathrm{F}$ decreased throughout gestation and reached the lowest values in the adults (Table 1). Mean corpuscular hemoglobin concentration was similar in all five groups.

An overview of the RBC deformation-shear stress results for the five groups is presented in Figure 2. RBC from the fetuses, the preterm infants, the term neonates, and the adults showed the same increase in elongation (i.e. deformation) with increasing shear stress (Fig. 2). At none of the applied shear stresses were significant differences in RBC deformation among the five groups observed.

The frequency distributions of RBC deformation determined for the five groups were almost symmetric (Fig. 3). The distributions for the neonates showed higher peaks and slightly broader pattern compared to the adults. Figure 3 shows that neonatal blood contains larger numbers of both poorly and extremely well deformable RBC.

Direct microscopic observation of RBC subjected to shear forces showed rotation of the membrane around the cell content ("tank-treading"). More than $95 \%$ of RBC from the fetuses, the preterm, and term neonates and the adults were capable of tanktread motion.
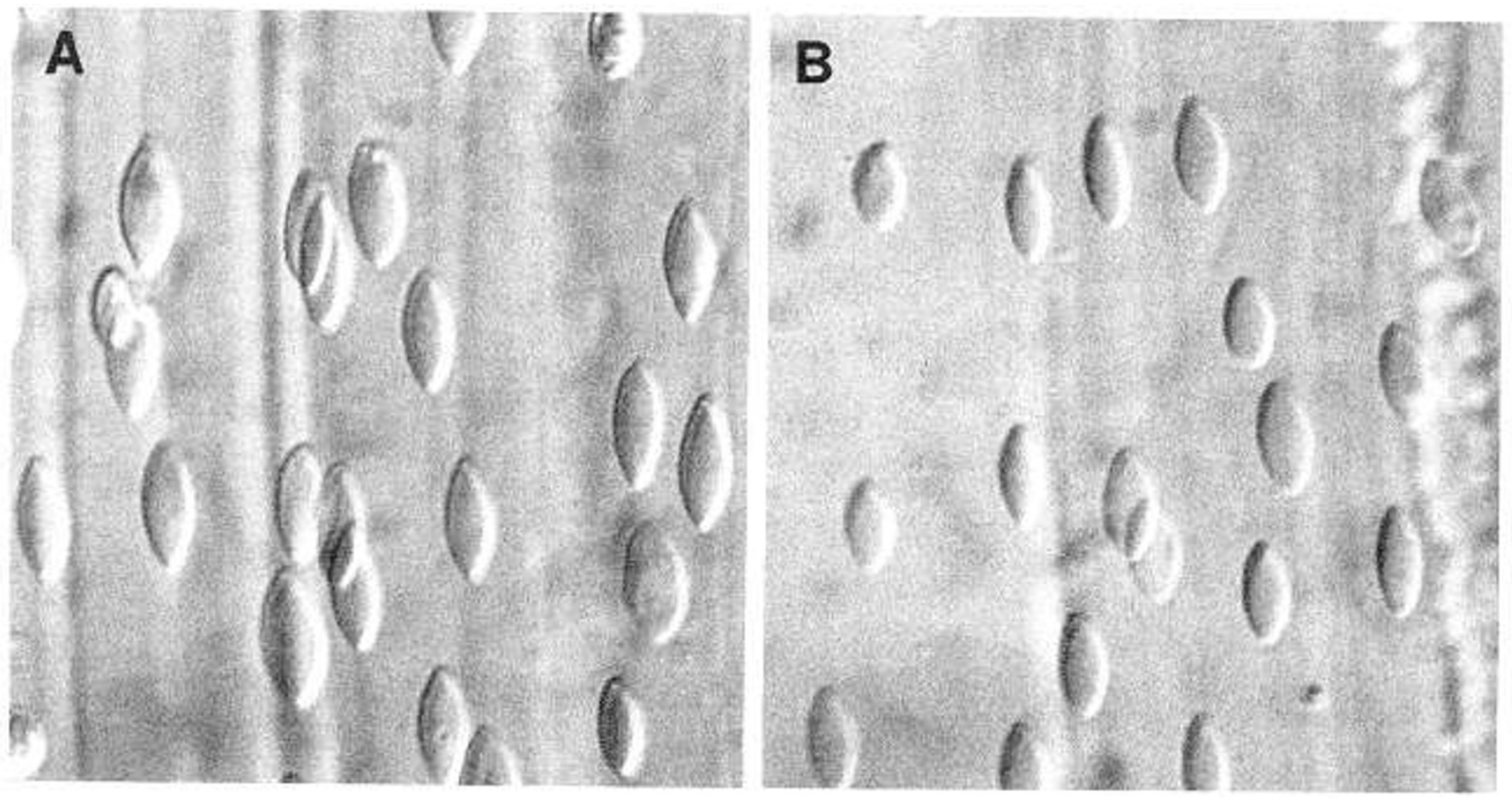

Fig. 1. Deformation of $\mathrm{RBC}$ in the rheoscope. The cells were suspended in 21 centipoise isotonic dextran solution and subjected to a shear stress of $85 \mathrm{dyn} / \mathrm{cm}^{2}$. Left panel, RBC from a fetus with $19 \mathrm{wk}$ of gestational age; right panel, adult RBC. Both cell types showed an average deformation index of 0.42. "Tank-tread" motion of the membrane around the cell content can be monitored by observing the motion of small membrane irregularities which are visible on some of the cells. 


\section{DISCUSSION}

This study demonstrates that the deformability of the average fetal and neonatal RBC is not different from the average adult $\mathrm{RBC}$ measured with the rheoscope. These results are not contradictory to the reports of decreased filterability of neonatal RBC (13-16) because the impaired filterability may be due to small subpopulations of poorly deformable (Fig. 3) and of very large RBC $(10,21)$ in neonatal blood. These cells could obstruct filter pores, thereby precluding the passage of subsequent RBC. Large cells have to deform more than small cells to enter narrow channels and are, therefore, more likely to obstruct their entrances $(12,16)$. This may explain why the larger $\mathrm{RBC}$ from

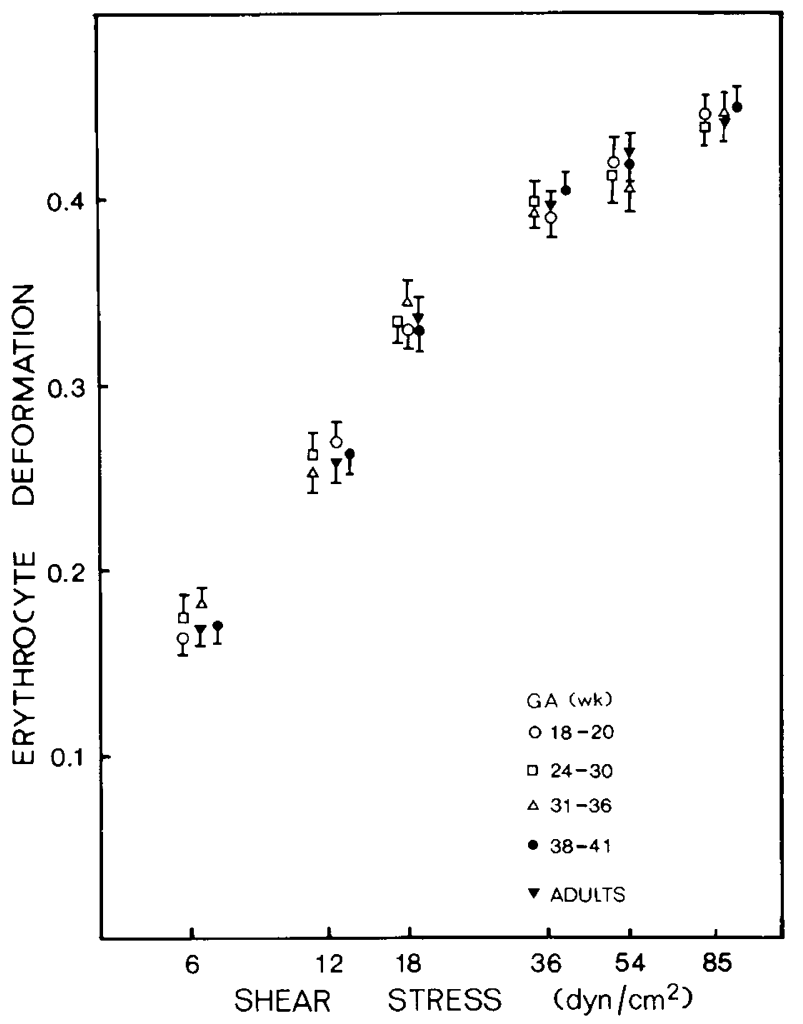

Fig. 2. Deformation of RBC from five fetuses, 20 preterm infants, 20 term neonates, and 20 adults plotted against shear stress. Erythrocyte deformation is defined as: $\mathrm{D}=(\mathrm{L}-\mathrm{W}) /(\mathrm{L}+\mathrm{W})$, where $\mathrm{L}$ is the length and $\mathrm{W}$ the width of the cells. Values represent mean $\pm \mathrm{SD}$. Note that $\mathrm{SD}$ was calculated from the sample means. There was no significant difference in RBC deformation among the five groups at any shear stress. preterm infants are less filterable than $\mathrm{RBC}$ from term neonates (15).

Cellular deformability is determined by extrinsic properties (overall cellular shape and excess surface area) and by intrinsic material properties (resistance of the membrane to elastic deformation and membrane and hemoglobin viscosity) $(2,12,22)$. $\mathrm{RBC}$ deformability as determined by the rheoscope is influenced by all of these factors $(2,10)$. These determinants have been studied for RBC from full-term neonates. Although neonatal $\mathrm{RBC}$ are markedly larger than adult RBC (Table 1), both cell types have similar overall shape and the same excess surface area of $39 \%$ beyond that required to enclose the cellular volume (21). Moreover, neonatal and adult RBC have similar membrane and hemoglobin viscosity $(12,16)$. Coulombel et al. (11) observed increased susceptibility of neonatal RBC to echinocytic transformation. However, we did not observe a greater tendency of fetal and neonatal RBC to morphological abnormalities in the rheoscope.

The static resistance of neonatal $\mathrm{RBC}$ membrane to elastic deformation is slightly decreased compared to adult RBC (12).

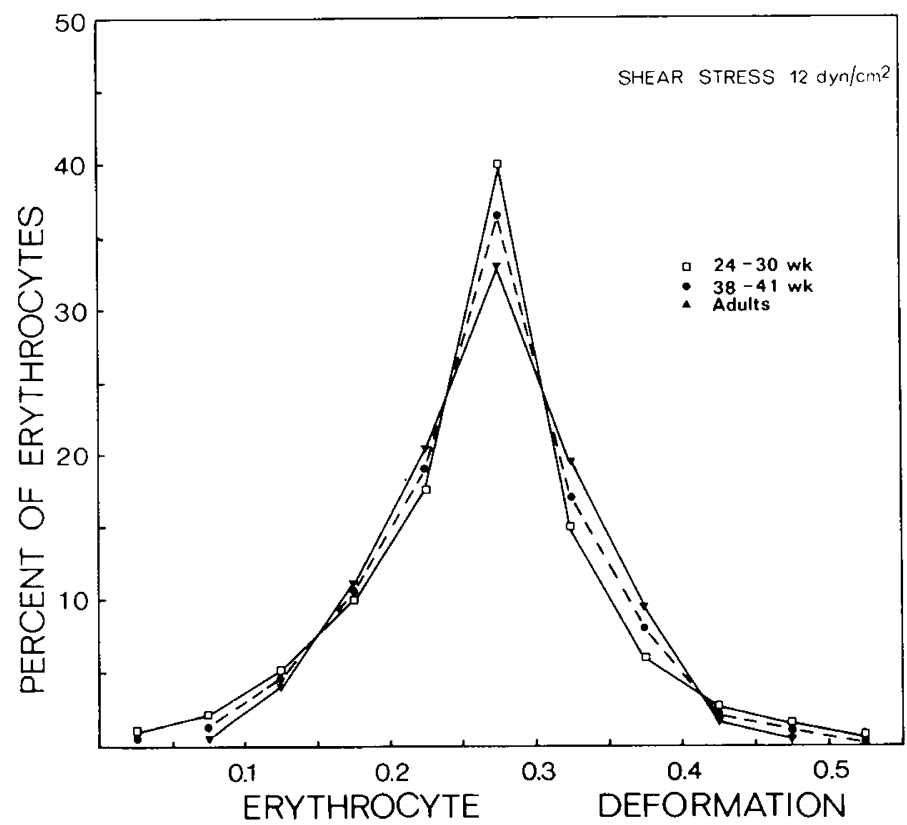

Fig. 3. Distribution of RBC deformability in preterm infants, term neonates, and adults. Distributions are based on studies of $400 \mathrm{RBC}$ in each group (i.e. $40 \mathrm{RBC}$ from each of the 10 donors). Note that neonatal $\mathrm{RBC}$ show higher peaks and slightly broader patterns compared to adult RBC.

Table 1. Hematological and deformability data for $R B C$ from fetuses $(a)$, preterm infants $(b, c)$, term neonates $(d)$, and adults $(e) *$

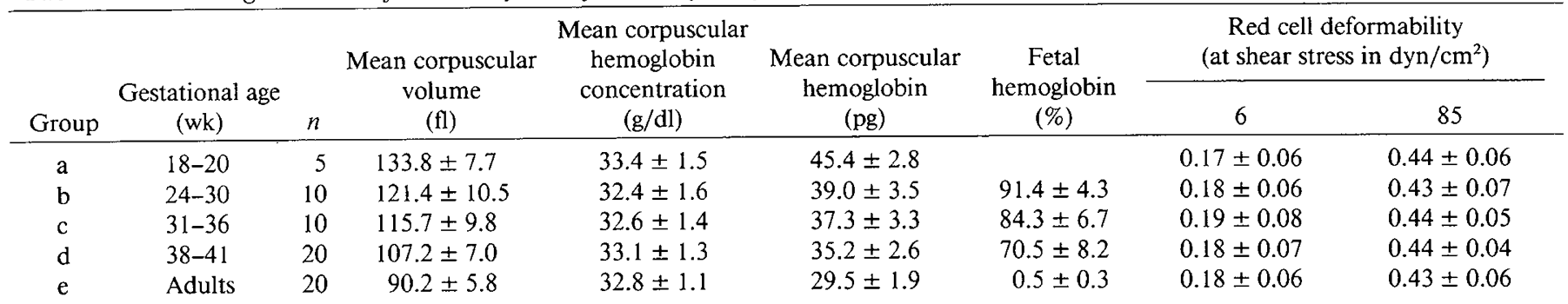

Significant differ$a>b=c>d>e \quad a=b=c=d=e \quad a>b=c=d>e \quad b>c>d>e \quad a=b=c=d=e \quad a=b=c=d=e$ ences among groups a-e $(\mathrm{P}<$ $0.05)$

* Values represent mean $\pm 1 \mathrm{SD}$. Note that the $\mathrm{SD}$ for the deformability data reflects the spread of the entire RBC populations in each group, whereas the SD values in Figure 2 have been calculated from the sample means. 
Determinants of RBC deformability have not been studied for $\mathrm{RBC}$ from fetuses or preterm infants. However, our finding of similar deformability of RBC from fetuses, preterm, and term infants suggests that these determinants of RBC deformability are also similar.

Deformation of $\mathrm{RBC}$ in the rheoscope is associated with membrane rotation around the cell content $(1,2,18)$. This "tanktread" motion was observed in the present study for fetal, neonatal, and adult RBC. Tank-treading is an important response of the cell to tangential shear forces because it enables the transmission of shear forces into the cytoplasm and allows rapid shape changes. Tank-treading occurs in narrow capillaries as well as in large vessels $(1,2)$.

Deformability of RBC is a major determinant of blood viscosity which in turn is very important for effective blood flow in the entire circulatory system $(1,2)$. Other determinants of blood viscosity are the hematocrit, RBC aggregation, and plasma viscosity. Recent studies have shown that both RBC aggregation and plasma viscosity are very low in preterm infants and increase with increasing gestational age because of the concomitant increase in plasma protein $(23,24)$. Normal deformability of the average RBC and diminished RBC aggregation and plasma viscosity explain why whole blood viscosity measured at given hematocrit and shear stress is lower in preterm infants than in term neonates and adults (23). However, the presence of a higher percentage of poorly deformable RBC in blood from preterm infants (Fig. 3) may suggest that preterm infants are more prone to pathological changes in RBC deformability (e.g. as a result of endotoxin and vitamin $\mathrm{E}$ deficiency) $(25,26)$. This may contribute to the susceptibility of preterm infants to serious complications associated with disturbed microcirculation (e.g. necrotizing enterocolitis) (6).

The life span of RBC increases with gestational age and reaches the highest value in adults (7). Thus, the average RBC in preterm infants is younger than the average adult RBC. RBC deformability is inversely related to RBC age $(10,18)$. Thus, similar deformability of neonatal and adult RBC implies decreased deformability of neonatal RBC for their age. The presence of a larger percentage of well deformable (and thus probably young) $\mathrm{RBC}$ in neonatal blood agrees with the shorter life span of neonatal RBC. The larger percentage of poorly deformable RBC agrees with the assumption of an accelerated aging process of neonatal RBC (10).

During the physiological aging process $\mathrm{RBC}$ lose membrane fragments and water leading to a decrease in volume and surface area and an increase in density and mean corpuscular hemoglobin concentration $(10,22)$. These alterations cause an age-dependent decrease in cellular deformability (10). Aging RBC in term neonates show an accelerated loss in volume, membrane surface, and deformability compared to adult RBC (10). A recent study suggests that the accelerated aging process of neonatal RBC is in part the consequence of decreased resistance of the membrane to yield in response to a given force, and to impaired ability of the large neonatal RBC to pass the narrow splenic slits (12), thereby facilitating fragmentation by immunological mechanisms.

Blood from preterm infants contains larger numbers of both poorly and highly deformable RBC than blood from term neonates (Fig. 3). This agrees with the finding that the life span of $\mathrm{RBC}$ in preterm infants is shorter than in term neonates (7). Furthermore, it suggests that the aging of RBC in the fetus is even more accelerated than in term neonates. Further studies on deformability and mechanical properties of age-separated RBC from fetuses or preterm infants are necessary to verify the hypothesis of accelerated RBC aging.

Acknowledgments. The authors thank Mrs. W. Himmel and her midwife colleagues for their cooperation and Mrs. L. Krüger for her secretarial assistance.

\section{REFERENCES}

1. Schmid-Schönbein H 1975 Erythrocyte rheology and the optimization of mass transport in the microcirculation. Blood Cells 1:285-306

2. Schmid-Schönbein H, Gaehtgens P 1981 What is red cell deformability? Scand J Clin Lab Invest 41(suppl 156):13-26

3. Kon K, Maeda N, Shiga T 1983 The influence of deformation of transformed erythrocytes during flow on the rate of oxygen release. J Physiol 339:573584

4. Canham PB, Potter RF, Woo D 1984 Geometric accomodation between the dimensions of erythrocytes and the calibre of heart and muscle capillaries in the rat. J Physiol 347:697-712

5. Cokelet GR 1981 Dynamics of erythrocyte motion in filtration tests and in vivo flow. Scand J Clin Lab Invest 41(suppl 156):77-82

6. Harrison MW, Connell RS, Campbell JR, Webb MC 1975 Microcirculatory changes in the gastrointestinal tract of the hypoxic puppy: an electron microscope study. J Pediatr Surg 10:599-607

7. Pearson HA 1967 Life-span of the fetal red blood cell. J Pediatr 70:166-171

8. Cohen RS, Ostrander CR, Cowan BE, Stevens GB, Hopper AO Stevenson DK 1982 Pulmonary excretion rates of carbon monoxide using a modified technique: differences between premature and full-term infants. Biol Neonate 41:289-293

9. Landaw SA 1978 Decreased survival and altered membrane properties of red blood cells (RBC) in the newborn rat. Pediatr Res 70:395(abstr)

10. Linderkamp O, Wu PYK, Meiselman HJ 1982 Deformability of density separated red blood cells in normal newborn infants and adults. Pediatr Res 16:964-968

11. Coulombel L, Tchernie G, Feo C, Mohandas N 1982: Echinocytic sensitivity and deformability of human newborn red cells. Biol Neonate 42:284-290

12. Linderkamp O, Nash G, Wu PYK, Meiselman HJ 1985 Deformability and intrinsic material properties of neonatal red blood cells. Blood (in press)

13. Gross GP, Hathaway WE 1972 Fetal erythrocyte deformability. Pediatr Res 6:593-599

14. Tillmann W, Zabel U, Lakomek M, Schröter W 1981 Influence of the fluidity of hemoglobin $F$ on the flexibility of red cells of newborn and older infants. Bibl Anat 20:222-225

15. Buchan PC 1981 Fetal haemorheology in normal pregnancy. In: 2nd European Conference on Clinical Haemorheology. London (abstr 127)

16. Reinhart WH, Danoff SJ, King RG, Chien S 1985 Rheology of fetal and maternal blood. Pediatr Res 19:147-153

17. Nash GB, Meiselman HJ 1983 Effects of dextran and polyvinylpyrrolidone on red cell geometry and membrane elasticity. Ann NY Acad Sci 416:255-262

18. Sutera SP, Gardner RA, Boylan CW, Carroll GL, Chang KC, Marvel JS, Kilo C, Gonen B, Williamson JR 1985 Age-related changes in deformability of human erythrocytes. Blood 65:275-282

19. Betke K, Marti H, Schlicht I 1959 Estimation of small percentage of foetal haemoglobin. Nature 184:1877-1878

20. Campbell RC 1967 Statistics for Biologists. Cambridge University Press, London

21. Linderkamp O, Wu PYK, Meiselman HJ 1983 Geometry of neonatal and adult red blood cells. Pediatr Res 17:250-253

22. Linderkamp O, Meiselman HJ 1982: Geometric, osmotic, and membrane mechanical properties of density-separated human red cells. Blood 59:11211127

23. Linderkamp O, Versmold HT, Riegel KP, Betke K 1984 Contributions of red cells and plasma to blood viscosity in preterm and full-term infants and adults. Pediatrics 74:45-51

24. Linderkamp O, Ozanne P, Wu PYK, Meiselman HJ 1984 Red blood cell aggregation in preterm and term neonates and adults. Pediatr Res 18:13561360

25. Linderkamp O, Klose HJ, Betke K, Fuhrmann G, Galanos C, Kelson S, Marget W, Riegel KP 1980 Effect of neonatal sepsis, endotoxin and exchange transfusion on the deformability of red blood cells. Pediatr Res 62:1424(abstr)

26. Lubin B, Chiu D 1982 Properties of vitamin E-deficient erythrocytes following peroxidant injury. Pediatr Res 16:928-932 